\title{
Erratum to: PET scanning in plastic and reconstructive surgery
}

\author{
Eirini Liodaki • Emmanouil Liodakis • \\ Othonas Papadopoulos · Hans-Günther Machens • \\ Nikolaos A. Papadopulos
}

Published online: 17 March 2012

(C) The Japanese Society of Nuclear Medicine 2012

\section{Erratum to: Ann Nucl Med (2012) 26:115-122 \\ DOI 10.1007/s12149-011-0557-0}

Errors occurred in designating the authors' forenames and surnames. The names of the authors should be shown as follows:

Surname: Liodaki, Forename: Eirini; Surname: Liodakis, Forename: Emmanouil; Surname: Papadopoulos, Forename: Othonas; Surname: Machens, Forename: Hans-Günther; Surname: Papadopulos, Forename: Nikolaos A.

The online version of the original article can be found under doi:10.1007/s12149-011-0557-0.

E. Liodaki ( $\square)$

Department of Surgery, University Hospital Mannheim,

University of Heidelberg, Theodor-Kutzer Ufer 1-3,

Mannheim, Germany

e-mail: liodaki_irene@yahoo.com

E. Liodakis

Trauma Department, Hannover Medical School,

Hannover, Germany

O. Papadopoulos

Department of Plastic and Reconstructive Surgery,

Laikon Hospital, University of Athens, Athens, Greece

H.-G. Machens · N. A. Papadopulos

Department of Plastic Surgery and Hand Surgery,

University Hospital Rechts der Isar,

Munich Technical University, Munich, Germany 